\title{
Effect of dielectric thin films on reflection properties of metal hole arrays
}

\author{
Fumiaki Miyamaru, ${ }^{1,2, a)}$ Yuki Sasagawa, ${ }^{1}$ and Mitsuo Wada Takeda ${ }^{1}$ \\ ${ }^{1}$ Department of Physics, Faculty of Science, Shinshu University, 3-1-1 Asahi, Matsumoto, \\ Nagano 390-8621, Japan \\ ${ }^{2}$ PRESTO, Japan Science and Technology Agency, 2-1-1 Katahira, Aoba, Sendai 980-0845, Japan
}

(Received 20 November 2009; accepted 21 December 2009; published online 12 January 2010)

\begin{abstract}
We study the effect of a dielectric film attached to the surface of a metal hole array (MHA) on the reflection spectrum in the terahertz $(\mathrm{THz})$ region. The frequency of the reflection dip, attributed to the excitation of surface waves in the vicinity of the MHA surface, shifts to lower frequencies with increasing dielectric film thickness. This resonant characteristic of MHAs can be applied to highly sensitive $\mathrm{THz}$ sensing for samples attached to the MHA surface. We also investigate the dependence of the reflection spectrum on the MHA's thickness and the side to which the dielectric film is attached. (c) 2010 American Institute of Physics. [doi:10.1063/1.3292024]
\end{abstract}

Recent remarkable developments in terahertz $(\mathrm{THz})$ technology have opened the way to new applications in sensing technology. Several practical applications have been proposed and demonstrated so far, such as medical diagnostics, ${ }^{1,2}$ security applications, ${ }^{3-5}$ and drug inspection. ${ }^{6}$ These sensing applications occupy an important place in $\mathrm{THz}$ technological research because many substances have distinct electromagnetic features in the $\mathrm{THz}$ region, allowing their detection and identification. In particular, highly sensitive detection of extremely small amounts of substances or small variations in a sample's condition is crucial and has the potential to advance the development of label-free sensing of biomolecules or gases.

Electromagnetic resonant structures are useful for highly sensitive detection and sensing. ${ }^{7,8}$ The sensitivity of sample detection could be increased significantly around the resonant frequency, realizing the detection of very small amounts of sample. In a previous study, we used metal hole arrays (MHAs), which are metal films perforated periodically with circular holes, as surface wave (SW) resonant sensors in the THz region. ${ }^{9}, 10$ By illuminating an MHA, a SW can be excited on the metal surface at a certain resonant frequency as a result of the interference of light scattered from the aperture array. ${ }^{11,12}$ Due to strong localization of the electromagnetic field of SWs in the vicinity of the MHA surface, the resonant transmission characteristics depend strongly on the dielectric distribution near the surface. ${ }^{3}$ This characteristic allows us to achieve highly sensitive detection of small amounts of sample placed at a subwavelength distance from the MHA surface. ${ }^{9,10}$

In our previous study, we applied this highly sensitive sensing to transmission measurement. In this study, for the purpose of highly sensitive sensing with MHAs by reflection measurement, we investigate the effect of a dielectric film attached to the MHA surface on the reflection spectrum of the MHA. Reflection measurement has an advantage over transmission measurement for $\mathrm{THz}$ sensing; i.e., electromagnetically opaque substances are available as detected samples.

The MHAs used in our study are 100 and $300 \mu \mathrm{m}$ thick metal films perforated with a triangular lattice structure of

${ }^{a)}$ Electronic mail: miyamaru@ @hinshu-u.ac.jp. circular holes, as shown in the inset of Fig. 1. For both MHAs, the diameter of the holes is $500 \mu \mathrm{m}$ and the period of the triangular lattice is $1000 \mu \mathrm{m}$. The reflection spectra of the MHAs, at normal incidence, are measured by a $\mathrm{THz}$ time-domain spectroscopic system that allows us to measure the waveform of the $\mathrm{THz}$ wave directly in the time domain. Using a Fourier transformation, we can calculate the reflection and phase spectra from the time-domain waveforms reflected by the MHAs and by a silver mirror used as a reference.

We first measured the reflection spectrum of a bare MHA with a thickness of $t_{\mathrm{MHA}}=100 \mu \mathrm{m}$ at normal incidence as shown in Fig. 1 (red line). A clear resonant dip is observed at $0.32 \mathrm{THz}$. The resonant frequency $f_{\mathrm{SW}}$ of the SW excited on the surface of the periodic structure is calculated from $^{14}$

$$
f_{\mathrm{SW}}=\left|\mathbf{k}_{\mathrm{in}}+\mathbf{G}\right| \frac{c}{2 \pi}\left(\frac{\varepsilon_{\mathrm{m}}+\varepsilon_{\mathrm{d}}}{\varepsilon_{\mathrm{m}} \varepsilon_{\mathrm{d}}}\right)^{1 / 2},
$$

where $\mathbf{k}_{\text {in }}$ is the in-plane wave vector of the incident electromagnetic wave, $\mathbf{G}$ is the reciprocal lattice vector, and $\varepsilon_{\mathrm{m}}$ and $\varepsilon_{\mathrm{d}}$ are the dielectric constants of the metal and the attached dielectric, respectively. From this equation, $f_{\mathrm{SW}}$ is estimated to be $0.34 \mathrm{THz}$ for the bare MHA $\left(\varepsilon_{\mathrm{m}} \gg \varepsilon_{\mathrm{d}}=1\right)$ at normal incidence $\left(\left|\mathbf{k}_{\text {in }}+\mathbf{G}\right|=2 / \sqrt{3}\right)$, which is higher than the observed resonant dip frequency. This discrepancy is attributed to Fano-like interference ${ }^{11,12}$ between the directly reflected

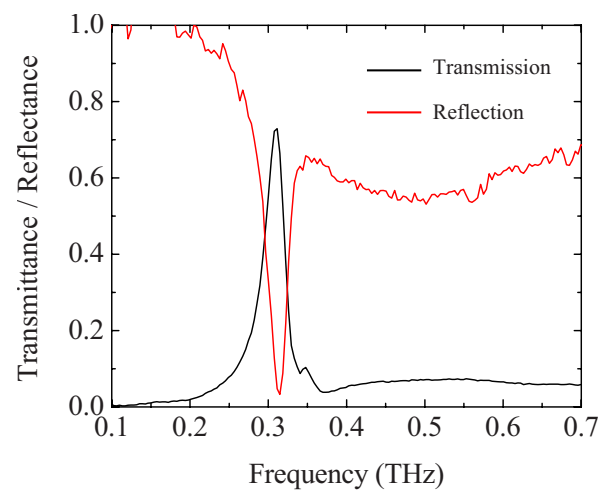

FIG. 1. (Color online) Measured reflection (red line) and transmission (black line) spectra for the $100 \mu \mathrm{m}$ thick MHA. 


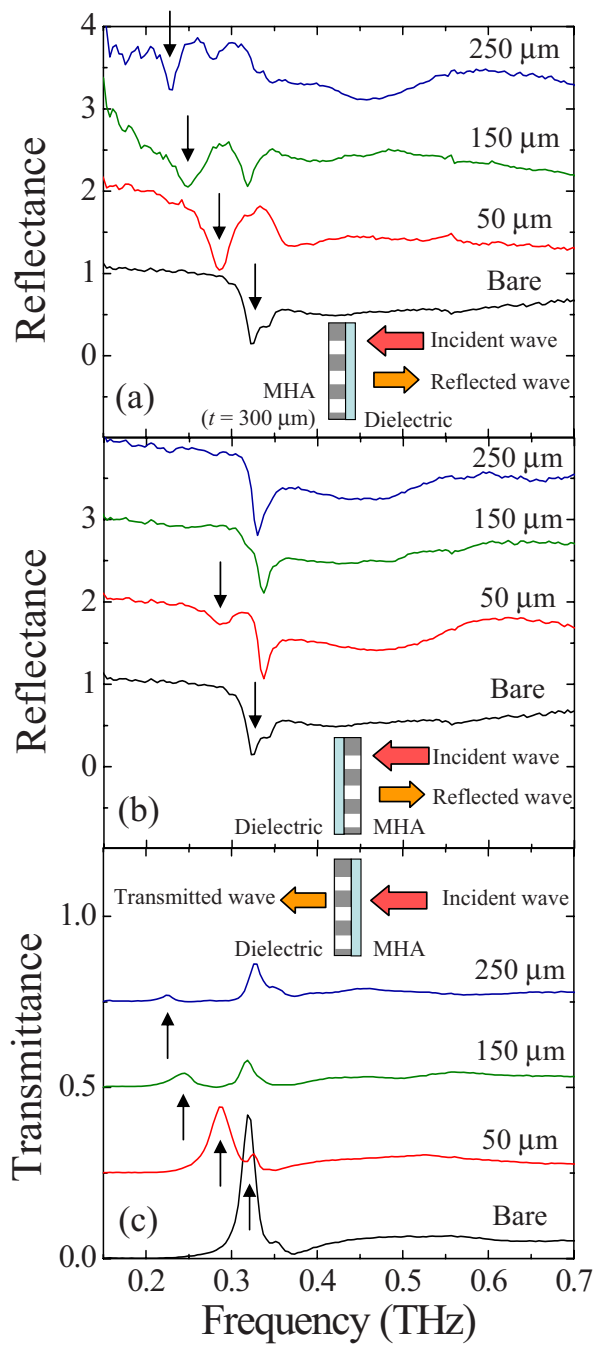

FIG. 2. (Color online) Measured reflection spectra for the $300 \mu \mathrm{m}$ thick MHA with PP films of several thicknesses $t_{\mathrm{PP}}$ attached to the (a) illuminated and (b) opposite sides of the array. (c) Measured transmission spectra for the same sample as (a). Schematic pictures of the optical configuration are shown in each figure.

wave and a reemitted wave from the SW excited near the metal surface. For comparison, we also measured the transmission spectra for the same MHA, shown in the black line of Fig. 1. The peak transmission frequency coincides well with the reflection dip frequency. This provides evidence that the resonant reflection dip, as well as the resonant transmission peak, is attributed to SW excitation. ${ }^{13}$ This also means that highly sensitive THz sensing with MHAs may be applicable to reflection-type measurement.

For the purpose of highly sensitive $\mathrm{THz}$ sensing application of reflection measurement, we investigate the effect of a thin film attached to one side of the MHA surface on the reflection characteristics. In this experiment, we used polypropylene (PP) films; the measured permittivity is about $\varepsilon_{\mathrm{PP}} \sim 2.9$ over the frequency range of interest. Figures 2(a) and 2(b) show the reflection spectra of the $300 \mu \mathrm{m}$ thick MHA with a PP film attached on the illuminated and opposite sides of the MHA, respectively. In Fig. 2(a), a reflection dip is observed for the bare MHA (black line) at $0.32 \mathrm{THz}$. The reflection dip frequency shifts to $0.29 \mathrm{THz}$ with a $50 \mu \mathrm{m}$ thick PP film attached (red line). With increasing PP film thickness $t_{\mathrm{PP}}$, the dip frequency decreases. For a PP film of $t_{\mathrm{PP}}=250 \mu \mathrm{m}$, the reflection dip is observed at $0.23 \mathrm{THz}$.
This dependence of the reflection dip frequency on the attached film thickness coincides with that observed in transmission measurement. ${ }^{13}$

In the application of highly sensitive $\mathrm{THz}$ sensing, reflection measurement has an advantage over transmission measurement. Figure 2(c) shows transmission spectra of the same samples as in Fig. 2(a). In the transmission spectra, the same frequency shift of the transmission peak with PP film thickness is observed as in the reflection spectra [Fig. 2(a)]. However, the peak transmittance decreases significantly with PP film thickness. This is due to the cutoff effect of the metal holes and the mismatch of the resonant frequency between the two sides. ${ }^{13}$ In particular, the cutoff effect is crucial for transmission measurement. The cutoff frequency $f_{\mathrm{c}}$ is estimated to be $0.35 \mathrm{THz}$ for our MHAs, which is higher than the highest peak frequency observed in Fig. 2(c). Below the cutoff frequency, an electromagnetic wave inside a metal hole is in evanescent mode, and the amplitude decreases exponentially as the electromagnetic wave propagates. The cutoff effect becomes stronger for lower frequencies; therefore, the transmission peak decreases significantly. Such a significant decrease in peak transmittance makes it difficult to measure the resonant peak with enough signal-to-noise ratio $(\mathrm{S} / \mathrm{N}$ ratio); thus, the sensitivity of $\mathrm{THz}$ sensing decreases considerably. In reflection measurement, on the other hand, the dip reflectance does not change so much as the PP film thickness increases [Fig. 2(a)]. This is because the cutoff effect is small in the reflection spectra. For reflection measurement, the information on the illuminated surface of the MHA contributes mainly to the reflection spectrum. When we put the PP film on the illuminated surface, the reduction in dip reflectance due to the cutoff effect can be avoided, leading to resonant dip measurement with a high $\mathrm{S} / \mathrm{N}$ ratio even if a thick PP film is attached.

When the PP film is attached to the opposite surface [Fig. 2(b)], the reflection dip becomes very weak for a PP film of $t_{\mathrm{PP}}=50 \mu \mathrm{m}$ (red line) and almost disappears for $t_{\mathrm{PP}}$ $=150 \mu \mathrm{m}$ (green line) and $250 \mu \mathrm{m}$ (blue line). The reflection dip, which is attributed to SW excitation on the metal-air interface of the illuminated surface, is observed at $0.32 \mathrm{THz}$ and is almost independent of the PP film thickness. This is because the contribution from the opposite surface to the reflection spectrum is small compared with that from the illuminated surface. An electromagnetic wave reflected at the opposite surface propagates through the metal hole twice; thus, its amplitude decreases more significantly compared to the case of transmission measurement. This is a disadvantage of reflection measurement for the application of highly sensitive $\mathrm{THz}$ sensing. Such a disadvantage can be resolved if a thinner MHA is used. Figures 3(a) and 3(b) show the reflection spectra of the $100 \mu \mathrm{m}$ thick MHA with a PP film attached to the illuminated and opposite sides, respectively. For the case of the PP film attached to the illuminated surface [Fig. 3(a)], the overall reflection spectra for all PP film thicknesses are quite similar to those in Fig. 2(a). On the other hand, for the PP film attached on the opposite surface [Fig. 3(b)], the reflection spectra are different from those in Fig. 2(b). Reflection dips are clearly observed even for the PP film of $t_{\mathrm{PP}}=250 \mu \mathrm{m}$. For thin MHAs $\left(t_{\mathrm{MHA}}=100 \mu \mathrm{m}\right)$, the contribution of electromagnetic waves reflected at the opposite surface to the reflection spectrum is large compared to the case of thick MHAs $\left(t_{\mathrm{MHA}}=300 \mu \mathrm{m}\right)$. This reflection 


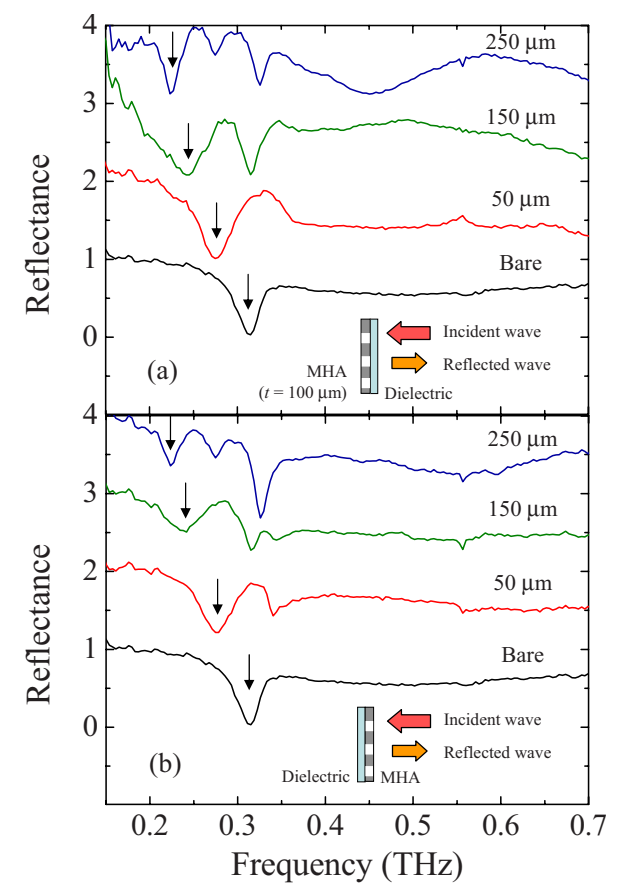

FIG. 3. (Color online) Measured reflection spectra for the $100 \mu \mathrm{m}$ thick MHA with PP films of several thicknesses $t_{\mathrm{PP}}$ attached to the (a) illuminated and (b) opposite sides of the array. Schematic pictures of the optical configuration are shown in each figure.

characteristic of the thin MHA can be applied to highly sensitive $\mathrm{THz}$ sensing of the surface condition of opaque samples, which cannot be measured by transmission measurement.

In summary, we investigated the effect of a dielectric film attached to an MHA surface on the reflection characteristics in the $\mathrm{THz}$ region. The reflection dip, which is attributed to SW excitation, shifts to lower frequencies with increasing thickness of a PP film attached to the MHA surface.
Even for a thick MHA, the dip reflectance is almost unchanged with variation in PP film thickness, indicating that resonant sensing with a high $\mathrm{S} / \mathrm{N}$ ratio is possible. We believe that these results can be applied to reflection-type highly sensitive $\mathrm{THz}$ sensing.

This work was partly supported by the Ministry of Education, Science, Sports and Culture, Grant-in-Aid for Scientific Research Grant No. 20244047, and for Young Scientists Grant No. 21760036. F.M. is also supported by the Murata Science Foundation and the Konica Minolta Imaging Science Foundation.

${ }^{1}$ K. J. Siebert, H. Quast, R. Leonhardt, T. Löffler, M. Thomson, T. Bauwe, H. G. Roskos, and S. Czasch, Appl. Phys. Lett. 80, 3003 (2002).

${ }^{2}$ S. Nakajima, H. Hoshina, M. Yamashita, C. Otani, and N. Miyoshi, Appl. Phys. Lett. 90, 041102 (2007).

${ }^{3}$ K. Yamamoto, M. Yamaguchi, F. Miyamaru, M. Tani, and M. Hangyo, Jpn. J. Appl. Phys., Part 2 43, L414 (2004).

${ }^{4}$ N. Karpowicz, H. Zhong, C. Zhang, K.-I. Lin, J.-S. Hwang, J. Xu, and X.-C. Zhang, Appl. Phys. Lett. 86, 054105 (2005).

${ }^{5}$ H.-B. Liu, Y. Chen, G. J. Bastiaans, and X.-C. Zhang, Opt. Express 14, 415 (2006).

${ }^{6}$ K. Kawase, Y. Ogawa, Y. Watanabe, and H. Inoue, Opt. Express 11, 2549 (2003).

${ }^{7}$ M. Nagel, P. H. Bolivar, M. Brucherseifer, H. Kurz, A. Bosserhoff, and R. Büttner, Appl. Phys. Lett. 80, 154 (2002).

${ }^{8}$ C. Rau, G. Torosyan, R. Beigang, and Kh. Nerkararyan, Appl. Phys. Lett. 86, 211119 (2005).

${ }^{9}$ F. Miyamaru, S. Hayashi, C. Otani, K. Kawase, Y. Ogawa, H. Yoshida, and E. Kato, Opt. Lett. 31, 1118 (2006).

${ }^{10}$ F. Miyamaru, M. W. Takeda, T. Suzuki, and C. Otani, Opt. Express 15, 14804 (2007).

${ }^{11}$ M. Sarrazin, J.-P. Vigneron, and J.-M. Vigoureux, Phys. Rev. B 67, 085415 (2003).

${ }^{12}$ C. Genet, M. P. van Exter, and J. P. Woerdman, Opt. Commun. 225, 331 (2003).

${ }^{13}$ M. Tanaka, F. Miyamaru, M. Hangyo, T. Tanaka, M. Akazawa, and E. Sano, Opt. Lett. 30, 1210 (2005).

${ }^{14} \mathrm{H}$. Raether, Surface Plasmons on Smooth and Rough Surfaces and on Gratings (Springer, Berlin, 1988). 